\title{
Biliary Ascariasis with Hepatic Abscess in a Young Boy of 2.5 Years of Age
}

\author{
MOHIBUR RAHMAN ${ }^{1}$, ROBED AMI N², TANVIR HOSSAI N ${ }^{3}$, KAZI ARI FUR RAHMAN ${ }^{4}$, HAZZRA SHARMI N ${ }^{5}$, AHMED \\ AHSAN HABIB ${ }^{6}$
}

\begin{abstract}
:
Ascariasis is the most widespread helminthiasis worldwide. In endemic areas, Ascaris lumbricoides infestation is a very widespread cause of biliopancreatic disease and one of common cause of pancreatitis. Liver abscess as a part of complication of biliary ascariais is possible but not common. Endoscopic management of biliopancreatic ascariasis is feasible, with a very low morbidity and a high rate of success. We experienced a paediatric patient of biliary ascariasis who developed liver abscess as part of complication and initial presentation.
\end{abstract}

Keyword : Helminthiasis, Ascariasis, Hepatic, Abscess

\section{Introduction:}

Ascaris lumbricoides is the most common nematode found in the human gastrointestinal tract with a greater prevalence found in developing tropical and subtropical countries. Most cases of ascariasis follow a benign course. In some cases the adult parasite can invade the biliary or pancreatic ducts and cause obstruction with development of cholecystitis, cholangitis, pancreatitis, and hepatic abscesses. ${ }^{1,2}$ Biliary Ascariasis is rare but is the commonest extra-intestinal complication. ${ }^{3}$ Early diagnosis and treatment is important to prevent these complications. ${ }^{2}$

Endoscopic retrograde cholangiopancreatography (ERCP) has a well-defined role in the management of biliary and pancreatic disorders in adults. However, its use in children is limited to a few centers in the West. Reasons for its limited use in children are: (i) diseases of the pancreas and biliary tract are less frequent in children than adults; (ii) until recently, paediatric instruments were not available; and (iii) the lack of skilled paediatric gastroenterologists in this technique. ${ }^{4}$

Moreover, in a developing country its use is further restricted because of the lack of awareness about its use, and fear of complications. ${ }^{4}$

We present here a $2 \frac{1}{2} 2$-year-old boy with biliary ascariasis with hepatic abscess, whose main complaint was abdominal pain and high grade fever. After ERCP, multiple round worms were extracted from the common bile duct (CBD). We report this case because paediatric liver abscess due to biliary ascariasis have rarely been reported.

\section{Case Report:}

A 2.5 year-old boy, hailing from a village in Dhaka, was admitted in the department of Paediatric Medicine on June 6 of 2009.

According to the mother he was quite well 15 days back since then he developed pain in right upper quadrant. The pain was severe and colicky in nature and used to subside spontaneously after 1-2 hours and there was no radiation. The mother also gave history of fever which was high grade, intermittent in nature and was associated with chills and rigor. Fever used to subside 1-2 hours after taking tablet paracetamol. She also mentioned that the boy passed worm with stool and vomitus during the period of illness. This child had no cough, shortness of breath, loose stool or increased urinary frequency. On examination the boy was found toxic, irritable, mildly anaemic, mildly dehydrated, not icteric, his pulse rate was 120 beats per minute, temperature$101^{\circ} \mathrm{F}$, respiratory rate-24 breaths per minute, body weight was $10 \mathrm{~kg}$. During per abdominal examination, the abdomen was found slightly distended, rigid, moderate to severely tender over the epigastric and right hypochondriac region. Bowel sound was normal. There was no organomegaly. Other systemic examinations were unremarkable. Laboratory investigations revealed- total count of WBC 15,100/cmm, poly morph 77\%, Lymphocyte-7\% Eosinophil-(15\%), Haemoglobin- $8.40 \mathrm{gm} / \mathrm{dL}$, ESR $80 \mathrm{~mm}$ in 1st hour. Serum bilirubin was $0.6 \mathrm{mg} / \mathrm{dL}$, ALT- $12 \mathrm{U} / \mathrm{L}$, Serum alkaline phosphtase $99 \mathrm{U} / \mathrm{L}$, serum amylase was $100 \mathrm{iu} / \mathrm{l}$. Stool routine and microscopic examination- revealed ova of Ascaris

1. Associate Professor, Department of Gastroenterology, Uttara Adhunik Medical College, Uttara, Dhaka-1230.

2. Assistant professor, Department of medicine, Dhaka Medical College, Dhaka

3. Medical Officer, Department of Gastroenterology, Uttara Adhunik Medical College, Uttara, Dhaka-1230.

4. Assistant Registrar. Department of Gastroenterology, Uttara Adhunik Medical College, Uttara, Dhaka-1230

5. Medical Officer, Department of Gastroenterology, Uttara Adhunik Medical College, Uttara, Dhaka-1230.

6. Medical Officer, Department of Gastroenterology, Uttara Adhunik Medical College, Uttara, Dhaka-1230.

Correspondence: Dr Mohammad mohibur Rahman, Associate Professor \& head, Department of Gastroenterology, Uttara Adhunik Medical College, Plot-34, Road-4, Sector-9, Uttara, Dhaka-1230.Email-rmohammadmohibur@yahoo.com 
Lumbricoides. Ultra sonogram of the abdomen showed dilated common bile duct with linear echogenic structures casting no acoustic shadow in the common bile duct and left hepatic duct with hepatomegaly and liver abscess. The patient was diagnosed as a case of Biliary Ascariasis with Liver Abscess. Patient was treated conservatively. But the condition of the patient did not improve. So Endoscopic retrograde cholangiopancreatography (ERCP) was performed under conscious sedation with injection midazolam on $13^{\text {th }}$ June 2009. ERCP was done by adult lateral viewing videoduodenoscope (Pentax ED-3470 TK) under fluoroscopic control. Instruments used during the endoscopic procedures were: Dormia basket (MTW), Wilson Cook extraction balloon (Medi-Globe, Germany), Microvasive sphincterotome. All instruments were re-usable as disposable materials were not available in our unit. Contrast agent was injected to check the patency of the ducts after extraction. During ERCP papilla looks inflamed and multiple dead round worms are seen protruding from the ampulla (figure 1, 2).

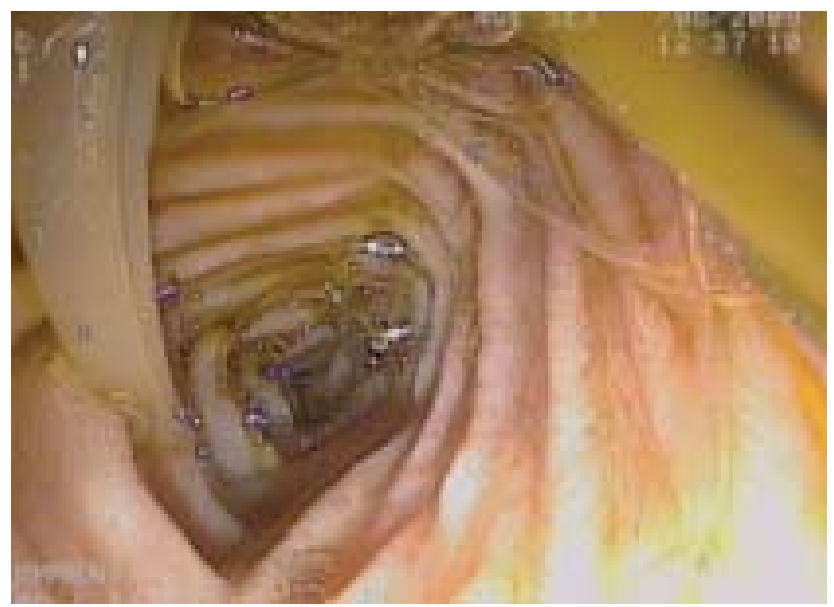

Fig.-1: Round worm seen in the $2^{\text {nd }}$ part of duodenum

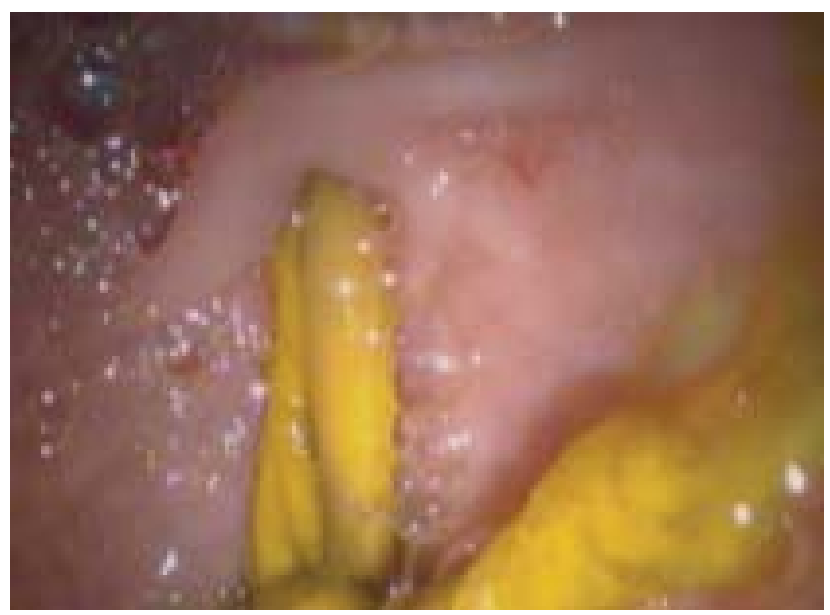

Fig.-2: Dead round worm impacted into the papiila.
Cholangiogram revealed dilated common bile duct, multiple tubular structures within the common bile duct. As the papilla was opened from the impacted round worm papillotomy was not done (figure 3). Multiple (seven) dead round worms extraction were done by the repeated dormia basket and balloon sweeping (figure 4). Subsequent

Cholangiogram showed no filling defect within common bile duct. Pancreatogram was not attempted. The procedure was uneventful. After extraction of worm by ERCP, the child started making dramatic recovery from the following day of ERCP as evidenced by subsidence of pain and fever. Patient was advised to take oral diet from the following day and syrup pyrental palmoate $200 \mathrm{mg}$ was given by mouth as anthelminthics. The patient was discharge on $16^{\text {th }}$ June with advised to continue antiobiotics as oral form and to come for follow-up. Follow-up ultra sonogram after one week revealed normal biliary tree and reduction in the size of the abscess. The patient was advised to take anthelminthics after every two months.

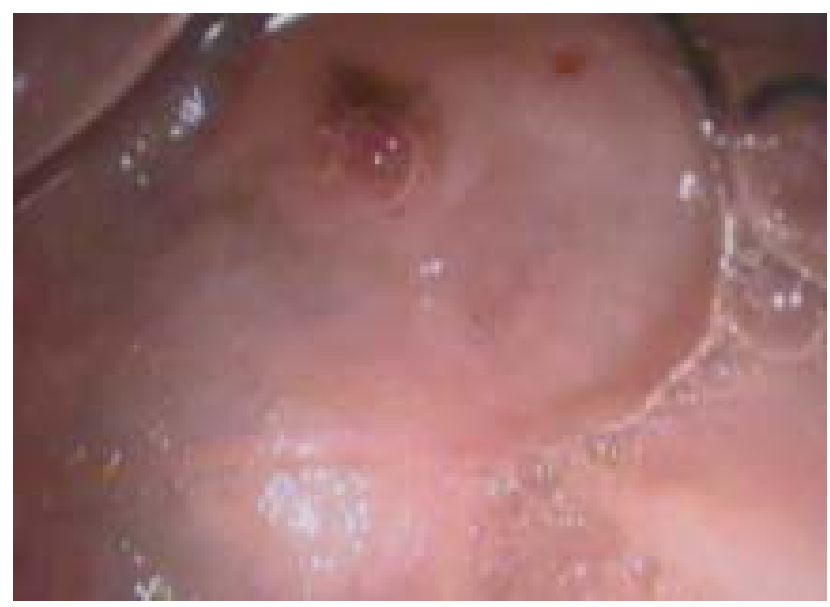

Fig.- 3: Opened and inflammed papilla after removal of round worm from the common bile duct

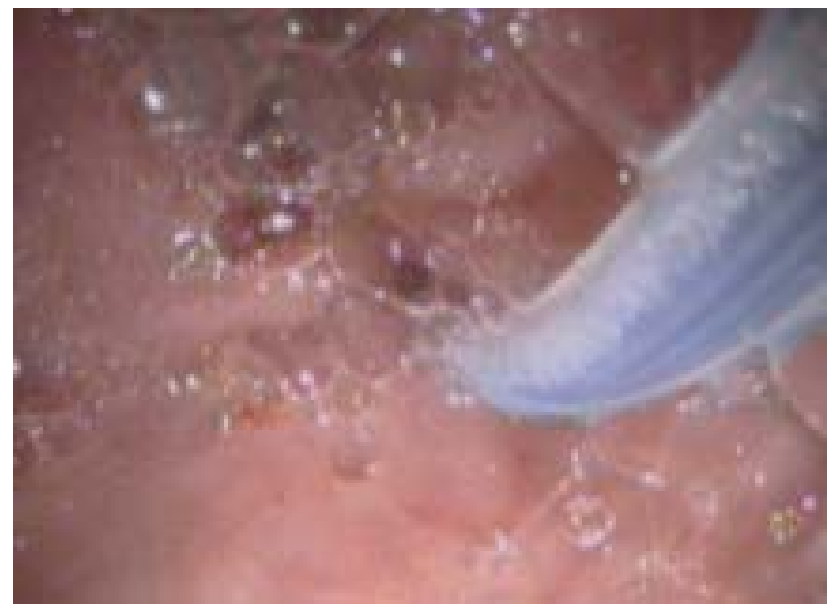

Fig.-4: Dormia basket in situ during removal of round worm from the common bile duct 
Endemic in tropical regions, the disease is caused by Ascaris lumbricoides, a nematode belonging to the Ascarides family, which infests the gut. Ascaris lumbricoides is a long milky white or pink roundworm with two eel-like ends. The adult male ascarsis measures 15 to $17 \mathrm{~cm}$ long and 2 to $3 \mathrm{~mm}$ in diameter. The oviparous adult female measures 20 to $25 \mathrm{~cm}$ long and 5 to $6 \mathrm{~mm}$ in diameter. ${ }^{5}$

Generally, the parasite does not cause overt clinical manifestations. Worm migration through viscera can however provoke mechanical obstruction of the intestine or direct toxic effects. Clinical manifestations may also result from bacteria born by the worm or a combination of these different effects. Intestinal problems are by far the most common. Biliopancreatic complications including ascending cholangitis, acute pancreatitis, acute cholecystitis, hepatic abscess, haemobilia, or intra hepatic lithiasis result from migration of the adult worm from the gut lumen into the biliary or pancreatic ducts via the ampulla of Vater. ${ }^{5}$ In some study, they found few cases of hepatic abscess from biliary ascariasis. 6,7

In endemic areas, Ascaris lumbricoides infestation is a very widespread cause of biliopancreatic disease and is responsible for nearly one-quarter of all cases of acute pancreatitis in endemic areas. Although most cases of biliopancreatic ascariasis are diagnosed in tropical regions, particularly in India, cases have also been reported from temperate regions and Africa. ${ }^{5}$

Though, children are commonly affected with round worm, biliopancreatic ascariasis in children is not common as it is difficult for the round worm to enter the biliary tree due to small size of the biliary tree and pancreatic duct. ${ }^{6,8}$ In a study conducted in Turkey, about $80 \%$ Children are above 10 years of age. One case was $11 / 2$ years old. ${ }^{3}$

Diffuse abdominal pain, often associated with fever and vomiting, is the predominant clinical symptom when the ascaris migrates into the biliary tree. Jaundice is observed in only $7 \%$ of the patients. ${ }^{5}$ Previous history of passage of worms in stool and vomitus may also present in some cases. ${ }^{3}$

Transparietal abdominal ultrasound is a simple, reliable, quick and inexpensive non invasive technique for the diagnosis and post therapeutic surveillance of biliopancreatic ascariasis. Typically, the longitudinal image of the biliary ascaris corresponds to a hyperechogenic tubular structure which may appear straight or curved, unique or multiple, producing a "rail track" image, or mobile within the dilated biliary duct, generally without a acoustic shadowing. The transverse image visualizes a round hyperechogenic structure with a hypoechogenic center. Likewise, in case of pancreatic ascariasis, the parasite may sometimes be visualized within the dilated duct. The transpapillary position of the worm cannot be determined ultrasonographically so the presence of part of the parasite in the duodenum may not be recognized. If part of the parasite is in the duodenum, it can be extracted with a standard endoscope which is generally more available than a lateral-viewing endoscope. ${ }^{5}$

Until recently, the diagnosis of ascariasis is often made at laparotomy or autopsy. Surgical management was the rule, which has a considerable impact on healthcare expenditures. ${ }^{5}$ More widespread use of endoscopic treatment has reduced the cost, both in terms of treatment and hospital stay. ${ }^{3,5}$ In suspected biliary tract disease, ERCP not only provides an accurate diagnosis, but also provides useful anatomical details that help to plan appropriate surgical intervention. ${ }^{4}$ But the role of endoscopic retrograde cholangiopancreatography (ERCP) is not yet fully established in children. ${ }^{4}$

Endoscopic retrograde catheterization of the biliary and pancreatic ducts is generally easy to achieve since the passage of the parasite through the sphincter of Oddi usually leaves the sphincter open. When the ascaris also lies in the duodenum, it can be extracted with a standard endoscope and a foreign body forceps under pharyngeal anesthesia. Inversely, if the parasite is entirely lodged in the duct, extraction of the mobile worm with a Dormia basket or a balloon is more difficult, often required general anesthesia for a longer procedure. ${ }^{5}$

Complications after endoscopic extraction of ascaris are exceptional. ${ }^{5}$ In a study conducted in Vietnam, they found only one case of acute edematous pancreatitis which resolved spontaneously among four patients who had biliary sphincterotomy. This low complication rate allows shorter hospitalization after endoscopic treatment (about 3 days) than after surgical treatment (about 12 days. The procedure is successful in most of the cases. ${ }^{5}$

Endoscopic retrograde cholangiopancreatography is commonly used for the diagnosis and treatment of pancreaticobiliary disorders in adults. The technique of ERCP is no more difficult in children than in adults, and there is no need for a special pediatric endoscope, except in infants younger than 12 months of age. The rate of successful cannulation of the desired duct in children is as good as in adults, and is reported to be in the range of 92-96\%. The complication rate of performing ERCP in children is reported to be less than that in adults. Therefore, ERCP can be performed safely and effectively in children. ${ }^{4}$

Anthelminthic chemotherapy is required to eradicate the parasites and prevent potentially serious complications. 
Mebendazole, albendazole and pyrantel pamoate are the most widely used agents to treat ascariasis. Preventive chemotherapy delivered to communities in endemic regions may serve as an affordable and cost-effective strategy to reduce the prevalence and morbidity in endemic regions. 9,10

\section{Conclusion:}

In obstructive biliary pathology, biliary ascariasis is a diagnosis that needs to be explored in our country. Knowledge of clinical symptoms, complications, and diagnostic and therapeutic options are of paramount importance for all health professionals in our country. Endoscopic management of biliopancreatic ascariasis is feasible, with a very low morbidity and a high rate of success. Endoscopic management of biliopancreatic ascariasis can be achieved easily in centers equipped with a lateral-viewing duodenoscope and adapted instruments. We have demonstrated that endoscopic treatment of biliopancreatic ascariasis is a highly successful procedure with low morbidity and zero mortality even in very young child. This method allows shorter hospital stay and minimal sequelae compared with surgical treatment. Endoscopic treatment is thus a very interesting option for this public health problem. Wider diffusion of this method would reduce hospital stays and costs.

\section{Conflict of Interest : None}

\section{References:}

1. De la Fuente-Lira M, Molotla-Xolalpa C, Rocha-Guevara ER. Biliary ascariasis. Case report and review of the literature. Cir Cir. 2006; 74:195-8.
2. Uysal G, Kösebalaban, Biliary ascariasis. Güven A.Indian J Pediatr. 2001 ; 68:1165-6.

3. Alam J, Wazir MD, Muhammad Z. Biliary ascariasis in children.J Ayub Med Coll Abbottabad. 2001; 13:32-3.

4. Poddar U, Thapa BR, Bhasin DK, Prasad A, Nagi B, Singh $\mathrm{K}$. Endoscopic retrograde cholangiopancreatography in the management of pancreaticobiliary disorders in children. $\mathrm{J}$ Gastroenterol Hepatol. 2001;16:927-31.

5. Mai Thi Hoi, Ariadne Desjeux, Thon Tat Bach, Marc Barthet, Jean-Charles Grimaud. Endoscopic management of biliary and pancreatic ascariasis in Viet-Nam Report of a series of 91 cases. Gastroentérologie Clinique et Biologique 2002; 26: 968-972.

6. Bahu M da G S, Baldisseroto M, Custodio C M, Gralha C Z, Mangili A R. Hepatobiliary and pancreatic complications of ascariasis in children: a study of seven cases. J Pediatr Gastroenterol Nutr 2001; 33: 271-5.)

7. Slesak G, Phanthavong P, Rasphone O, Luangxay K, Anoulakkham P, Pahatsalang V, Soumphonphakdy B, White JA, Newton PN. Obstructive biliary ascariasis with cholangitis and hepatic abscesses in Laos: a case report with gall bladder ultrasound video.J Infect. 2007; 54: e233-5. Epub 2007 Feb 20.

8. Khuroo M S, Ascariasis. Gastroenterol Clin North Am 1996; 25: 553-77.

9. Jennifer Keiser, Jürg Utzinger, Efficacy of Current Drugs Against Soil-Transmitted Helminth Infections- Systematic Review and Meta-analysis. JAMA. 2008;299:1937-1948.

10. St Georgiev V. Pharmacotherapy of ascariasis.Expert Opin Pharmacother. 2001; 2: 223-39. 